\title{
IMPROVING DIAGNOSIS PERFORMANCES ON A TRUCK ENGINE MAKING USE OF STATISTICAL CHARTS
}

\author{
Antoine Berton*, Erik Frisk ${ }^{* *}$ and \\ Mattias Nyberg ${ }^{* * *}$ \\ * Process Observation and Optimization Laboratory, \\ Université Laval, Québec, Canada G1K YP4. Email: \\ antoine.berton@gmn.ulaval.ca \\ ** Vehicular Systems, ISY, Linköping University, S-581 83 \\ Linköping, Sweden. Email: frisk@isy.liu.se \\ *** Scania CV AB, Södertälje, Sweden. Email: \\ matny@isy.liu.se
}

\begin{abstract}
A method to improve model based diagnosis for the air path of a truck engine is presented. Originally, inaccuracies in both the volumetric efficiency static model and sensors limited the diagnosis performances. Statistical charts built from truck operational data were tested in order to reduce the overall residual dispersion. Data taken from two different trucks working in various operating conditions were used to evaluate the proposed approach. The use of charts is improving the diagnosis performances by approximately $30 \%$. Copyright (c)2004 IFAC.
\end{abstract}

Keywords: Fault Detection, On-board Diagnosis, Diesel Engine, Residual, Dispersion Reduction.

\section{INTRODUCTION}

Since the nineties, as the environmental regulations became more strict, an increasing amount of work has been done to develop on-board diagnostics (OBD) system on car and truck engines. With the regulations of EURO-4 in 2005, truck manufacturers will have to include an OBD system in every truck. The purpose of such a system is to verify if environmental requirements on emissions are kept during the whole truck operational life.

In this paper, diagnosis of components in the air path is considered. It represents an important part of the diagnosis requirements for diesel engines. In this path, the potential sensor faults, actuator faults or leakages typically lead to degraded emission control. On one hand, the regulations ask to detect faults as soon as the emissions are affected. On the other hand, the truck manufac- turers want the level of false alarm to be very low. This opposite objectives require to optimize the fault detection procedure in order to bring it as accurate as possible.

The standard tools used in production vehicles (Unger and Smith, 1993; Jurgen, 1994) might not be sufficient to fulfill the requirements of the new regulations. One way to enhance the performance of fault diagnosis is to make use of more knowledge, by performing model-based fault diagnosis. For this study, the only available model of the engine is a basic static model, based on the engine volumetric efficiency (Heywood, 1988).

A previous work on engine diagnosis (Haraldsson, 2002) showed that using this raw model to generate residuals and perform fault detection is relatively inaccurate and motivates the development of more precise dynamic models. But the urgent 
need for a reliable engine diagnosis procedure justify the present attempt to optimize the diagnosis based on the static model. This is done by integrating statistical charts, that improve the static model accuracy.

\section{MODEL DESCRIPTION}

The engine of study in this paper is an experimental diesel engine with exhaust gas recirculation (EGR). A schematic view of the engine air path is shown on Figure 1. The air entering the engine

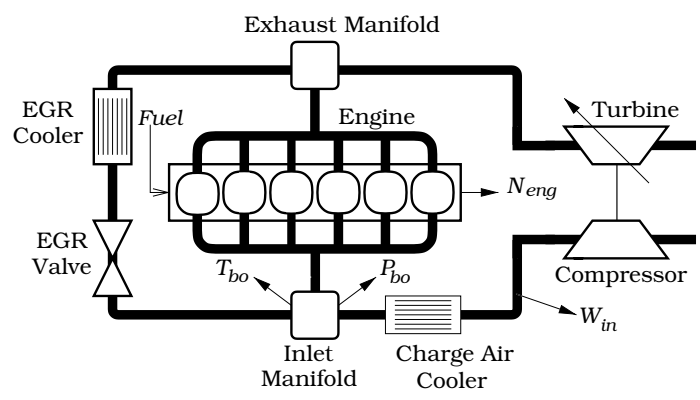

Fig. 1. Schematic overview of the Scania test engine.

is measured by an air-mass flow-meter and the corresponding measurements is noted $W_{i n}$. Then the air goes through a compressor and a charge air cooler, enters the intake manifold where it is mixed with exhaust gases, and flows into the cylinders where the fuel is added. The exhaust gases are divided one part to drive the turbine and another part to be recycled via the EGR. The involved measurements for residuals generation are the air-mass flow $\left(W_{i n}\right)$, the boost pressure $\left(P_{b o}\right)$ and boost temperature $\left(T_{b o}\right)$ at the inlet manifold, as well as the engine speed $\left(N_{\text {eng }}\right)$. Those measurements corresponds to the available sensors on the production version of the engine.

Studies are currently being made in order to accurately include the EGR path in the engine model. However, the present approved model does not allow to take into account the EGR component of the air path. Thus, the EGR valve was shut for all the experiments presented in this paper.

The model of the air path in the engine is based on a variable that measures the effectiveness of the engine induction: the volumetric efficiency. This model infers the air-mass flow in the inlet based on other available measurements. Equation 1 illustrates this predicted air-mass flow $\left(W_{p}\right)$.

$$
W_{p}=\frac{P_{b o} N_{e n g} n_{c y l} V_{d} \eta_{v o l}\left(P_{b o}, N_{e n g}\right)}{120 R T_{b o}}
$$

In Equation 1, $n_{c y l}$ is the number of cylinders, $V_{d}$ is the displacement volume of a cylinder and $R$ is the gas constant. The volumetric efficiency map $\left(\eta_{v o l}\right)$ depends on both boost pressure and engine speed and has to be calibrated from operation data. The air-mass flow is in $\mathrm{kg} / \mathrm{s}$, the engine speed is in rpm, the boost pressure is in Pascal and the boost temperature is in Kelvin. It is aimed in this paper to obtain the best diagnosis results as possible, using only this basic static model, instead of using a more complex dynamic model.

\section{DIAGNOSIS PROBLEM}

The diagnosis starts under predefined conditions. These conditions must be so defined that they appear in the standardized OBD test cycle. At a basic sampling rate of $20 \mathrm{~Hz}$, an average diagnosis sample could contain about 15000 measurements of each variable. Then, for each cycle, the test quantity for fault detection will be the average value of the residuals over this observation window.

\subsection{Residual Generation}

In this work, several hours of non-faulted data of the truck operation were available. Basically, those data were taken driving the truck in a private track at the Scania installation in Södertälje, Sweden. Many different atmospheric and road conditions were tested.

The natural candidate to be used as residual for fault detection is the difference between the measured and the predicted air-mass flow. Due to inaccuracy in both the model and the measurements, a significant bias remains in the mathematical expectancy of the residuals and must be handled. One of the dataset has been arbitrarily designated as the calibration set, as the others will be used for validation. This calibration set is used to evaluate both the volumetric efficiency map and the bias (mathematical expectancy of the raw residuals). Depending on which calibration set that is used to evaluate these parameters, the bias will be different. The general expression for residual calculation is:

$$
r=W_{i n}-W_{p}+b
$$

Taking many different samples of 15000 points from the calibration set, the bias $b$ has been evaluated to $0.0129 \mathrm{~kg} / \mathrm{s}$ for a sensor operating range of $0.05 \mathrm{~kg} / \mathrm{s}$ to $0.50 \mathrm{~kg} / \mathrm{s}$.

\subsection{Residual Distribution}

From this basic residual generation, it is interesting to plot the residuals in a histogram in order to see what is their approximate distribution. Figure 2 shows the distribution of the residual mean values of about 1000 different samples of 15000 points from the calibration set. As not so much data were available, the different samples 


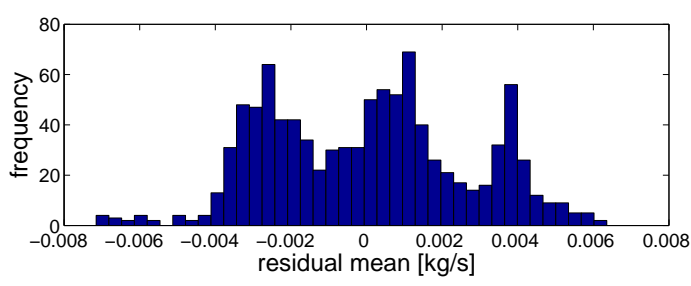

Fig. 2. Averaged residual distribution for the calibration set.

were overlapping in order to have a significant residual distribution population. From this only distribution, it could seem that faults in the range of $0.01 \mathrm{~kg} / \mathrm{s}$ could be easily detected. However, it does not agree with results of Figure 3 where the residual mean distributions for the calibration set as well as for five different validation sets are plotted. The first plot is the distribution of the calibration set residual mean values and the second and third plots are validation sets taken from the same truck, but in different conditions. The three last plots are validation sets taken from a second truck, similar to the first one.

Figure 3 illustrates the whole problem of fault detection in a real engine. The model calibrated with one dataset is not reliable when applied to other datasets. The biases observed in Figure 3 are significant as they appear to be higher than the
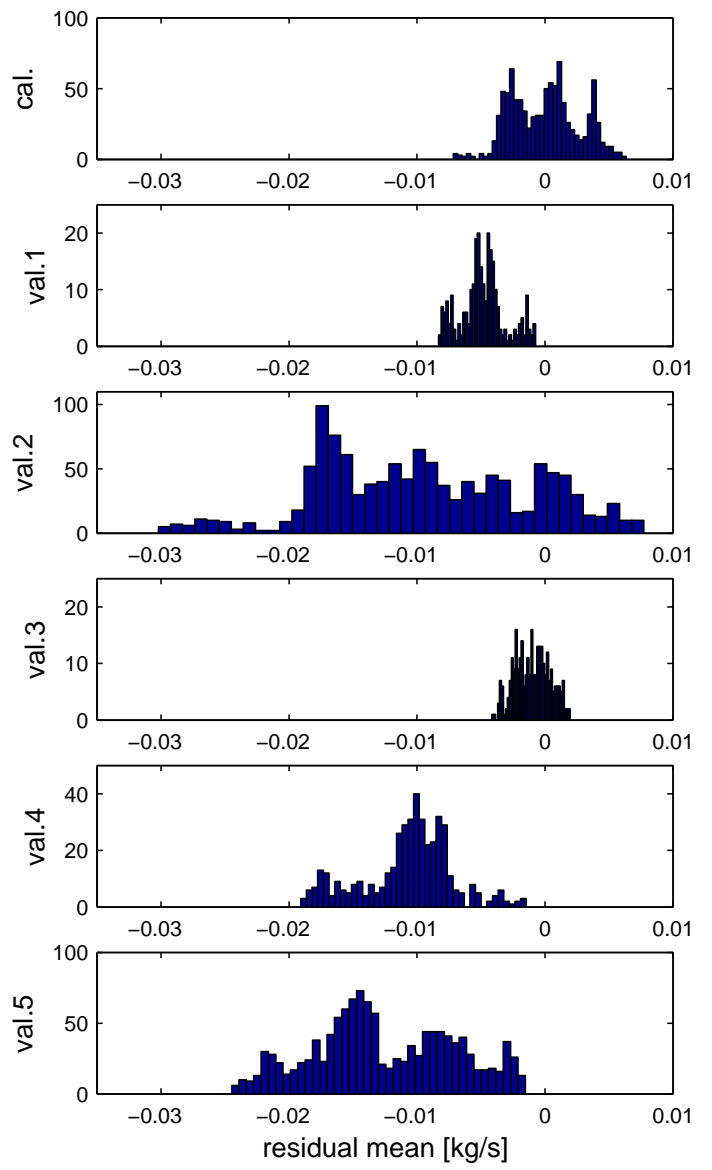

Fig. 3. Averaged residual distributions for the calibration set and five validation sets. dispersion of the original distribution. It would then be possible to detect fault only in the range of $0.03 \mathrm{~kg} / \mathrm{s}$. From that point, the work will consist in finding a way to make the overall residual generation more reliable.

\section{RESIDUAL CHARACTERIZATION}

If we intend to improve the residual informative content, it is necessary to study which variables have a strong influence on the residual values. Therefore plotting the raw residuals against different variables of interest could give an hint about the most influent ones. In the following, examples of plots of residuals against various variables of operation are presented in order to show this kind of interaction. The residuals are plotted with respect to a raw measurement, a measurement derivative and finally a linear combination of different variables.

\subsection{Plots Against Measurements}

From previous knowledge of the sensors and the volumetric efficiency model, it is already known that the air-mass flow level has an influence on both measurements and model accuracy, and therefore on the residual values. Figure 4 presents the raw residuals (without the bias correction of Equation 2) against the air-mass flow measurement. From Figure 4, it is obvious that the statis-

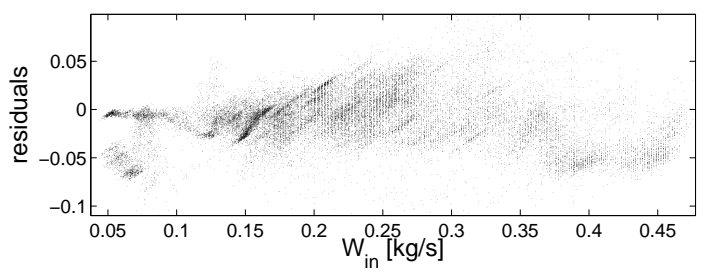

Fig. 4. Raw residuals of the calibration set against the air-mass flow measurement.

tical properties of the residuals are not constant with the air-mass flow level. Figure 5 shows the plot of both mean and standard deviation of the residuals with respect to $W_{i n}$.

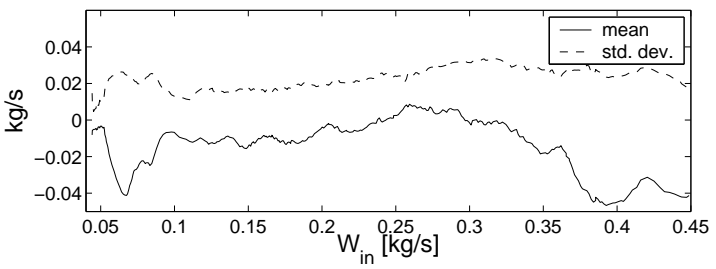

Fig. 5. Residual mean and standard deviation of the calibration set against the air-mass flow measurement. 


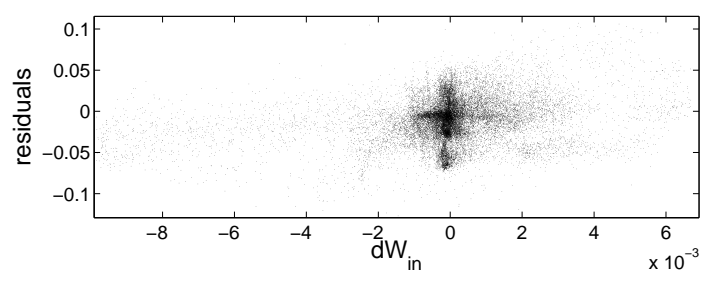

Fig. 6. Raw residuals of the calibration set against the air-mass flow derivative.

\subsection{Plots Against Measurements Derivative}

A priori, as the model is static and the truck is always changing of operating level, one can imagine that the measurement derivatives would be more appropriate to discriminate reliable residuals. Figure 6 shows the residuals plotted against the derivative of the air-mass flow. Even if it is probably not as evident as for the plot against $W_{i n}$, mean and variance are also varying with the air-mass flow derivative, as shown on Figure 7.

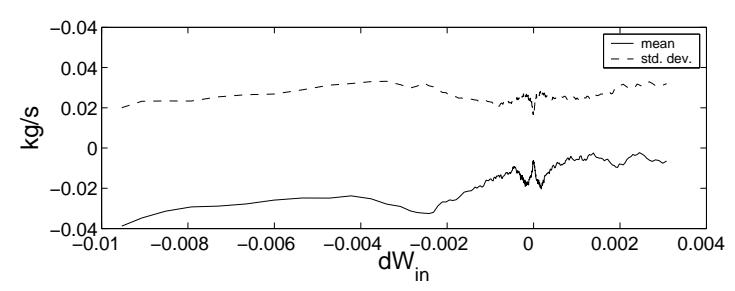

Fig. 7. Residual mean and standard deviation of the calibration set against the air-mass flow derivative.

\subsection{Plots Against Scores of PCA}

Plots of residuals can be made with respect to the four different measurements, as well as the four measurement derivatives. But it is also possible to plot the residuals against a linear combination of the different available variables. Doing so, the natural linear combination to be tested is the projection of the variables onto the principal components of the system (Wold et al., 1987).

Figure 8 shows the residuals against the scores of the first principal component of a PCA made over the four measurements and the four measurement derivatives ( 8 different variables). Around null scores, it seems that the residuals have a smaller dispersion and a mean closer to zero. This

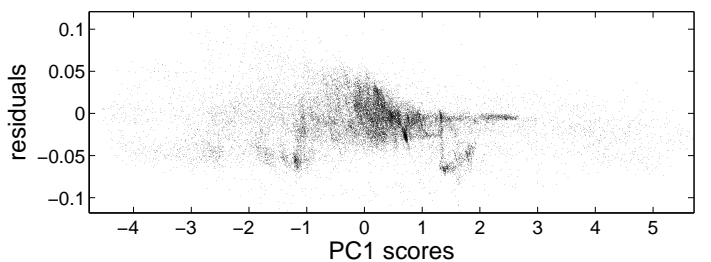

Fig. 8. Raw residuals of the calibration set against the scores of the 1st principal component.

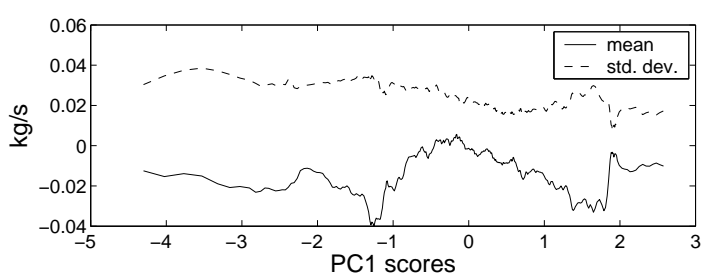

Fig. 9. Residual mean and standard deviation of the calibration set against the scores of the 1st principal component.

is shown on Figure 9 where the evolving mean and standard deviation are presented.

\subsection{Discussion on Residual Characterization}

The precedent figures were example of relations between the residuals and the variables of operation. Many other variables of operation are available to realize similar plots (Berton, 2003). The aim of this section was only to bring to the fore the evidence that additional information is available in those relations. This information (adaptive residual mean and standard deviation) can be used to generate more accurate residuals. In the next section, the various variables of operation on which the adaptive statistical properties can be based are assessed in order to find the one that improve the most the overall residual dispersion.

\section{EMPIRICAL MODEL IMPROVEMENT}

As statistical properties of the residuals depends on measurable level of operation of the truck engine, it is natural to try to use this additional information in order to improve the residual generation. First, the new method for residual generation is presented and then the potential variables which the statistical properties rely on are assessed.

\subsection{Improved Residual Generation}

In section 3 , the bias $b$ was an averaged bias and was the same for every residual of a sample. The main idea of the new residual generation is to use adaptive statistical properties for the generation of each residual among the 15000 of a cycle sample. In other words, depending on an operational variable to be determined, different bias (mean) and standard deviation will be assigned to each residual value.

Instead of using Equation 2, the residuals will then be generated by:

$$
r=\bar{\sigma} \frac{W_{i n}-W_{p}+b\left(x_{i}\right)}{\sigma\left(x_{i}\right)}
$$

In Equation 3, $b$ is a bias taken from a chart that depends on the observation $i$ of the operational 
variable $x$. In a same way, $\sigma$ is taken from a chart also depending on $x_{i}$. For example, those charts could be the ones of Figures 5, 7 or 9. Dividing by an adaptive standard deviation $\sigma$ aim to put a higher weight on the more accurate residuals. In order to bring back the original dimension of the residual, the quotient is multiplied by $\bar{\sigma}$, the average standard deviation over the whole sample. In that way, the resulting residual of Equation 3 will be comparable to the previous residual of Equation 2. Finally, the test quantity for fault detection will again be the mean of all the 15000 residuals of the sample.

\subsection{Chart Selection}

In Section 4, three different possible charts have been presented, but many other are available, as charts can be built on any operational variable, filtered variable, linear combination of variables, etc. It is then necessary to determine what chart would bring the larger improvement.

The main problem observed in Figure 3 is the evident difference in the distribution mean of the different datasets. A good indicator of a given chart performance would then be the difference between the calibration set residual distribution mean and the validation sets residual distribution means. Many different charts have been studied in order to find the one that minimizes this difference. Table 1 shows, for the six different datasets, the bias of the distribution mean with and without the use of adaptive charts. The charts studied were on the operational variables $W_{i n}$, the air-mass flow derivative $\left(d W_{i n}\right)$, the scores of the first principal component (PC1) and the scores of the second principal component (PC2). The last line of Table 1 shows the sum of the absolute biases for each chart.

\begin{tabular}{cccccc} 
data & no chart & $W_{i n}$ & $d W_{i n}$ & PC1 & PC2 \\
\hline cal. & 0.0000 & 0.0002 & 0.0002 & -0.0007 & 0.0004 \\
val.1 & -0.0048 & -0.0019 & -0.0049 & -0.0041 & -0.0016 \\
val.2 & -0.0095 & -0.0041 & -0.0098 & -0.0065 & -0.0058 \\
val.3 & -0.0010 & -0.0038 & -0.0018 & -0.0041 & 0.0011 \\
val.4 & -0.0107 & -0.0139 & -0.0116 & -0.0115 & -0.0075 \\
val.5 & -0.0126 & -0.0179 & -0.0136 & -0.0109 & -0.0089 \\
tot. & 0.0386 & 0.0418 & 0.0419 & 0.0378 & 0.0253 \\
\hline \multicolumn{5}{c}{ Table 1. Mean of averaged residual dis- } \\
tributions, for different datasets and \\
\multicolumn{5}{c}{ charts. }
\end{tabular}

The chart on $W_{\text {in }}$ shows really good results on the first two validation sets, which were taken from the same truck as the calibration set. But when it comes to validation sets taken from the second truck engine (3,4 and 5), the results are no more reliable. In Table 1 , the sum of the distribution absolute means show that the bias is generally minimized using a chart on the scores of the second principal component (PC2). Figure 10 illustrates the corresponding charts for residuals mean and standard deviation. This result is not

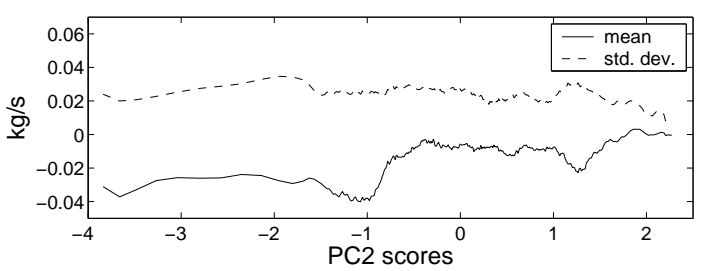

Fig. 10. Residual mean and standard deviation of the calibration set against the scores of the 2nd principal component.

an absolute one as not all the different chart alternatives have been studied. But among all the ones studied, this chart is the best candidate to improve residual reliability.

\section{DISPERSION REDUCTION OF THE RESIDUALS}

From the results of Table 1, it is already known that the use of the chart on $\mathrm{PC} 2$ will reduce the spreading of the different dataset residual distributions. Figure 11 shows exactly the same distributions as in Figure 3, but when the charts have been used to generate the residuals. Showing the same scale as Figure 3, Figure 11 demonstrates that the new residual distributions are less spread
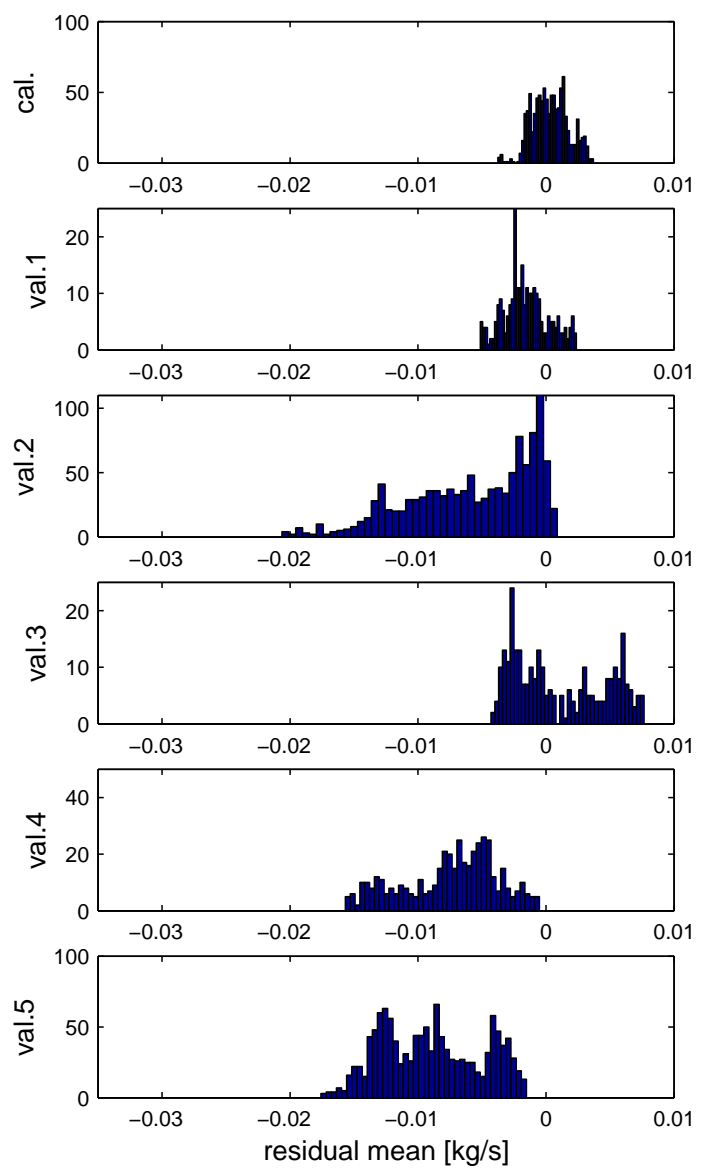

Fig. 11. Averaged residual distribution for the calibration set and five validation sets, when charts are used to generate residuals. 

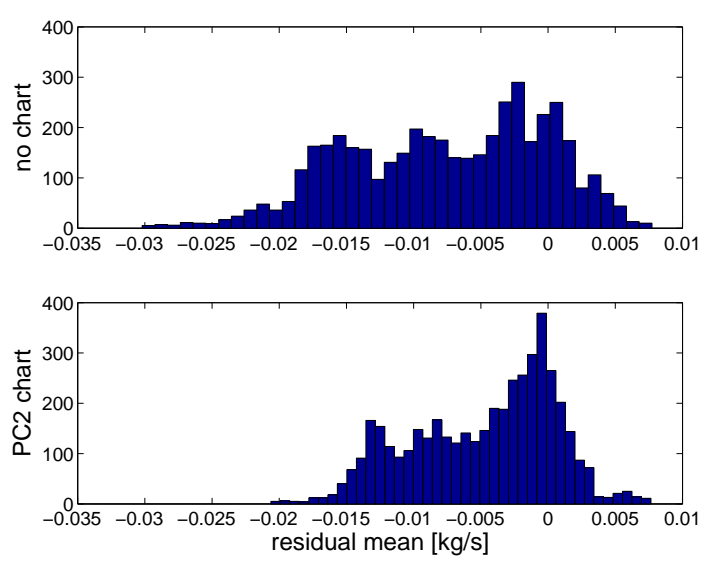

Fig. 12. Averaged residual distribution of all datasets, without chart and with a chart on PC2.

than the original ones. This is even more obvious in Figure 12, where the residuals from all data sets are added together. The first plot shows the original residuals and the second plot shows the residuals processed through the charts on PC2. The overall range of the distribution is reduced by approximately $20 \%$ and has its mean closer to zero.

\section{DIAGNOSIS IMPROVEMENT FROM CHARTS}

As the overall dispersion of the residuals is reduced making use of charts, the performances of a fault detection procedure should be enhanced. Figure 13 shows the detection test power function for a simulated sensor fault on the air-mass flow measurement. The power function shows the probability of detection of a fault depending on the fault amplitude (Casella and Berger, 1990). This power function has been roughly evaluated considering two different Gaussian distributions, on each sides of the two residual distributions of Figure 12, and setting the false alarm rate to 1 $\%$. Depending on which dataset has been used to calibrate the chart, the residual distributions as well as the power functions would be different. Ideally, a chart calibrated from a large amount of data could end in symmetrical residual distribution and power function. But for the illustration of the method, this was not necessary. With the available chart, Figure 13 shows that there is a significant gain in the detection performances. On

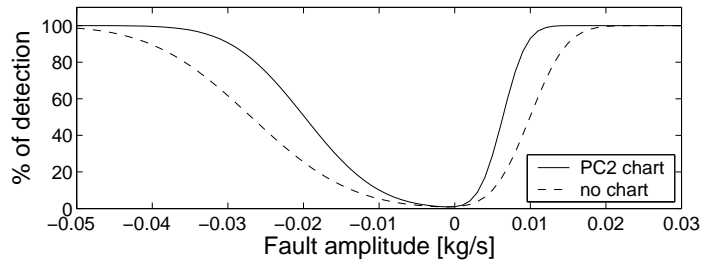

Fig. 13. Power functions evaluation for a $W_{i n}$ sensor bias. the positive side, the $\simeq 100 \%$ detection is obtained for faults of amplitude $0.013 \mathrm{~kg} / \mathrm{s}$ instead of 0.021 $\mathrm{kg} / \mathrm{s}$. For negative sensor biases, $\simeq 100 \%$ detection is obtained for faults of $0.037 \mathrm{~kg} / \mathrm{s}$ amplitude instead of more than $0.05 \mathrm{~kg} / \mathrm{s}$, without making use of a chart. Overall, this means about a $30 \%$ improvement in the size of detectable fault, for a similar false alarm rate.

\section{CONCLUSION}

With the incoming new regulations, the problem of diagnosis for truck engines need solutions in a near future. To match regulations, faults have to be detected as early as possible, without showing false alarm. Often, the available process models are not accurate. Therefore it is now necessary to deal with this lack of accuracy and try to improve model-based diagnosis with other tools.

Starting from an inaccurate model of the truck engine air path, this study proposed a way to generate residuals of the model using adaptive statistical properties (mean and standard deviation) taken from empirical charts. Doing so, the overall dispersion of the resulting residuals was significantly reduced compared to the original ones. This dispersion reduction in the residuals is improving the diagnosis performances by approximately 30 $\%$.

\section{ACKNOWLEDGEMENTS}

This work was supported by the Fonds québécois de la recherche sur la nature et les technologies.

\section{REFERENCES}

Berton, Antoine (2003). Improving Residual Generation for OBD in the Air Path of a Truck Engine. ISY Report (LiTH-R-2527). Linköpings Universitet, Sweden.

Casella, George and Roger L. Berger (1990). Statistical inference. Duxbury Press, Belmont, CA.

Haraldsson, Petter (2002). Optimisation of a diagnosis test for a truck engine. Master's thesis. ISY, Linköping Universitet.

Heywood, John B. (1988). Internal Combustion Engine Fundamentals. McGraw-Hill International.

Jurgen, Ronald (1994). Automotive Electronics Handbook. McGraw-Hill.

Unger, A. and K. Smith (1993). The OBDII system in the Volvo 850 turbo. $S A E P a-$ per.(932665).

Wold, S., K. Esbensen and P. Geladi (1987). Principal Component Analysis. Chemometrics and Intelligent Laboratory Systems (2), 3752. 